\section{Spaite et al Responds}

To the Editor:

We are elated that out article has stirred up such a "ruckus"! Although we could easily write ten pages in response to the many queries, we will deal succinctly with the major and pertinent issues.

1. We did not publish a condemnation, but a finding with profound implications. The pertinent flaws in the investigation are dealt with in the Limitations section of the article. According to the [preceding] Letters to the Editor, the fatal design flaws seem to have somehow made it through the expert and blinded reviewers of the journal. We readily concede that the study was not flawless; however, we all await with bated breath the first flawless EMS investigation.

2. After more than two decades of prehospital personnel responding to medical emergencies in the field, we still have very little understanding of what actually happens in the prehospital setting. Our investigation reveals a small piece of the picture in one state. However, this piece of the picture is extremely important, and we suspect it is relevant to more states than Arizona.

3. We strongly disagree that the potential biases in this study would have been decreased had the observer been a medically trained professional. In many of the responses, a medical observer would have been both ethically and medicolegally bound to become involved in the care of patients. This truly would have been a fatal flaw in the design since it would have introduced a direct alteration in the provision of prehospital care.

4. We agree that the incidence of omission of taking a pulse could not have been overestimated due to the observer not recording certain "non-traditional" means of estimating pulse rate. Because of this, we chose to deal almost entirely with the issue of blood pressure omission in the Discussion section. Somehow (because to us, it seems so obvious) we failed to emphasize the simplicity of the observation of the act of measuring a blood pressure. From our (perhaps naive) perspective, it does not take a "rocket scientist" to note and record whether a blood pressure cuff was actually placed on a patient's arm, pumped up, and followed by either auscultation or pal- pation of the pressure. However, in our defense, we would like to note that this undertaking was accomplished by a research technician who is a doctoral student in astrophysics (a real rocket scientist).

5. Each of the communications has raised a concern that some of the circumstances in which a blood pressure was omitted were perhaps settings in which the pressure either could not or should not be taken. We agree that such circumstances do exist and fall into primarily five categories: a) patients without a pulse; b) extremely short transport times to an appropriate facility (especially with critical patients); c) uncooperative or agitated patients; d) competent patients (or guardians) who refuse assessment when assessment is offered; or e) appropriate equipment is unavailable. The bulk of the concerns expressed in the communications to the editor fell into these categories. We do have the data regarding these issues and apologize for not reporting them in the paper; the data are: Among the 61 patients with blood pressure measurements omitted: a) four had no detectable pulse; b) one had a total treatment time (scene time plus transport time) of less than 15 minutes; c) two were unobtainable due to agitated patients; d) two occurred when competent patients refused the assessment despite it being offered; and e) three were small children in which pediatric BP cuffs were not available. Excluding these patients, 49 of 227 patients ( $21.6 \%$ ) had blood pressure measurements omitted without a good reason. This does decrease slightly the percentage of patients with blood pressure omission [from the initial percentage of $26.8 \%$ ]. However, we believe this still isl unacceptably high and it certainly does not alter the conclusions of our study.

After review of the concerns expressed by the previous correspondents, we still have shown that a significant number of patients could have and should have had their blood pressure taken in the field by ALS personnel in Arizona, but these measurements were not taken.

On a final note, each of the letters concluded that this issue certainly warrants further investigation in multiple settings and systems. We concur whole- heartedly, but find it puzzling that not a single letter noted the incredible difficulty of undertaking a study such as ours in the first place. A combination of: 1) expense; 2) garnering cooperation from 20 different EMS agencies; 3) the geo0graphically diverse setting; 4) finding a qualified person who was willing to travel throughout an entire state and respond into nearly any conceivable setting, 5) obtaining the cooperation and support of the state EMS authorities; and 6) to overcome the general reluctance that most people and agencies have of being evaluated by an entity which is largely unknown. These factors contributed to making this study a relatively monumental undertaking in and of itself. We can only hope that those who might attempt to repeat this study in other systems will not be stopped by the huge barriers that must be overcome simply to get off the ground.

Daniel W. Spaite, MD

Elizabeth A. Criss, RN

Terence D, Valenzuela, MD, FACEP

Harvey W. Meislin, MD, FACEP

Paul Hinsberg, BS

Section of Emergency Medicine

College of Medicine

The University of Arizona, Tucson, Arizona, USA

\section{Endotracheal Intubation}

To the Editor:

In 1971, I started teaching EMS to a classroom of firemen hungry to learn. In the beginning, there were moments when it was not certain who was teaching whom. Many of the procedures on which I was supposed to be educating them, they already had been exposed to, at a proficiency level superior to my medical training.

Still, there were some skills which I, as an educated and licensed emergency physician, could pass along to EMS personnel; skills they were more than eager to be taught.

Because it had such life-saving potential, the most important of these how-to, EMS procedures was endotracheal intubation.

Over the past two decades, more than fifteen-hundred EMS practitioners have been successfully instructed in endotracheal intubation at my hospital. Elsewhere, in Michigan and in scores of enlightened medical communities across the United States, thousands of other EMS providers have been properly 